\title{
The Convexity of a Fully Nonlinear Operator and Its Related Eigenvalue Problem
}

\section{Jiuzhou Huang*}

School of Mathematical Sciences, University of Science and Technology of China, Hefei 230026, P.R. China.

Received May 8, 2018; Accepted June 14, 2018

\begin{abstract}
We first get an existence and uniqueness result for a nonlinear eigenvalue problem. Then, we establish the constant rank theorem for the problem and use it to get a convexity property of the solution.
\end{abstract}

AMS subject classifications: 35J15, 35P30, 52A99

Key words: Hessian operator, eigenvalue problem, constant rank theorem, strict convexity.

\section{Introduction}

Let $\Omega \subset R^{3}$ be a strictly convex bounded domain in $R^{3}$ with smooth boundary. We consider the following eigenvalue problem

$$
\begin{cases}\sigma_{2}\left(W_{i j}\left(D^{2} u\right)\right)=\lambda(-u)^{2} & \text { in } \Omega, \\ u=0 & \text { on } \partial \Omega,\end{cases}
$$

where $D^{2} u=\left(u_{i j}\right)$ is the hessian matrix of $u,\left(W_{i j}\left(D^{2} u\right)\right)$ is a symmetric matrix defined as

$$
\left(W_{i j}\right)=\left[\begin{array}{ccc}
u_{11}+u_{22} & u_{32} & -u_{31} \\
u_{23} & u_{11}+u_{33} & u_{21} \\
-u_{13} & u_{12} & u_{22}+u_{33}
\end{array}\right]
$$

and $\sigma_{2}$ is the 2-nd hessian operator (i.e. $\sigma_{2}(S)=$ the sum of the 2-principal minors of $S$ for any $3 \times 3$ symmetric matrix $S$ ). We first prove an existence and uniqueness result for (1.1). Then we get some convexity result for the solution of it.

The eigenvalue problem played an important role in partial differential equations and had been studied by many authors (see, e.g., [21, 24, 25, 33]). Lions [25] first got

${ }^{*}$ Corresponding author. Email address: hjiuzhou@mail.ustc.edu.cn (J. Huang) 
the existence and uniqueness result for the eigenvalue problem of Monge-Ampère equation. Later on, Wang [33] (and Geng-Yu-Qu [12]) generalized this result to the k-hessian equations. In this paper, we get similar results for (1.1). Another important problem in PDE is the convexity problem, which connects the geometric properties to geometric inequalities. One powerful tool to study the convexity is the constant rank theorem. Caffarelli-Friedman [6] proved a constant rank theorem for convex solutions of quasilinear elliptic equations in $R^{2}$. Meanwhile, a similar result was discovered by Yau [30]. Korevaar-Lewis [23] generalized their results to $R^{n}$. Later on, Caffarelli-Guan-Ma [8] and Bian-Guan [4] established the constant rank theorem for a class of fully nonlinear equations. Related to our problems, Liu-Ma-Xu [26] established the constant rank theorem for the eigenvalue problem related to $k-H e s s i a n$ equations for $k=2$ in dimension $n=3$. In this paper, we would get a convexity result for (1.1) similar to [26].

Our another motivation to study (1.1) comes from the concept of $k$-convex solutions introduced by Harvey-Lawson [18] who introduced some general convexity on the solutions of the nonlinear elliptic Dirichlet problem. In their definition, a $C^{2}$ function $u$ is said to be $k$-convex if the sum of any $k$ eigenvalues of its hessian matrix is nonnegative. Recently, Han-Ma-Wu [16], Tosatti-Weinkove [31] studied a similar "convexity"-the $n-1$ plurisubharmonicity for $C^{2}$ functions defined on $\Omega \subset C^{n}$ (i.e. the sum of any $n-1$ eigenvalues of the complex hessian $\left(\frac{\partial^{2} u}{\partial z_{i} \partial \bar{z}_{j}}\right)$ is positive) and [31] used it to study the form-type Calabi-Yau equation (see $[10,11]$ ). The $k$-convexity is related to $(1.1)$ in the sense that if we note the eigenvalues of $D^{2} u$ by $\lambda_{i},(i=1,2,3)$. Then, by an orthogonal transformation, it is easy to know that the three eigenvalues of $\left(W_{i j}\left(D^{2} u\right)\right)$ are $\lambda_{1}+\lambda_{2}, \lambda_{1}+\lambda_{3}, \lambda_{2}+\lambda_{3}$. So, $u$ is 2 - convex if and only if $\left(W_{i j}\left(D^{2} u\right)\right)$ is positive semi-definite. For our purpose here, we do not need $\left(W_{i j}\left(D^{2} u\right)\right)$ to be positive semi-definite. Instead, we only need $\left(W_{i j}\left(D^{2} u\right)\right) \in \Gamma_{2}$ (the definition of $\Gamma_{2}$ will be given below) for the operator $F\left(D^{2} u\right)=\sigma_{2}\left(W_{i j}\left(D^{2} u\right)\right)$ to be elliptic on $u$.

We see that the operator $F\left(D^{2} u\right)=\sigma_{2}\left(W_{i j}\left(D^{2} u\right)\right)$ is a combination of the 2-hessian operator $\sigma_{2}$ and a linear one. In our proof of the theorems, we will use the elementary properties of the hessian operator repeatedly. So, let us state some preliminary knowledge that will be used below.

For $1 \leq k \leq n$, let $\sigma_{k}$ be the $k$-th elementary symmetric function, i.e.

$$
\sigma_{k}(\lambda)=\sum_{1 \leq i_{1}<i_{2}<\cdots<i_{k} \leq n} \lambda_{i_{1}} \lambda_{i_{2}} \cdots \lambda_{i_{k}}, \quad \forall \lambda=\left(\lambda_{1}, \ldots, \lambda_{n}\right) \in R^{n} .
$$

Let $S^{n}$ be the set of all $n \times n$ real symmetric matrix. For $S \in S^{n}$, let $\lambda(S)=\left(\lambda_{1}, \lambda_{2}, \ldots, \lambda_{n}\right)$ be the eigenvalues of $S$. We use the same notion $\sigma_{k}$ to define the $k$-hessian operator as

$$
\sigma_{k}(S)=\sigma_{k}(\lambda(S)) .
$$

We denote $\Gamma_{k}=\left\{\lambda \in R^{n} \mid \sigma_{i}(\lambda)>0, i=1, \ldots, k\right\}$, which is an open convex cone in $R^{n}$. We also denote $\Gamma_{k}=\left\{S \in S^{n} \mid \lambda(S) \in \Gamma_{k}\right\}=\left\{S \in S^{n} \mid \sigma_{i}(S)>0, i=1, \ldots, k\right\}$ if there is no confusion. It is well known that the $k$-hessian operator $\sigma_{k}$ is elliptic with respect to $S$ in $\Gamma_{k}$ and 
$\sigma_{k}^{\frac{1}{k}}$ is concave in $\Gamma_{k}$. For our use here, we only consider the $k=2$ case and we define the following concept of 2-admissible functions similar to that of Caffarelli-NirenbergSpruck [7].

Definition 1.1. Let $\Omega \subset R^{3}$ be a domain, a function $u \in C^{2}(\Omega)$ is said to be a 2 -admissible function in $\Omega$ if $\left(W_{i j}\left(D^{2} u\right)(x)\right) \in \Gamma_{2}$ for every $x \in \Omega$, where $\left(W_{i j}\left(D^{2} u\right)(x)\right)$ is defined as in (1.2). We denote the set of all $C^{2} 2$-admissible functions in $\Omega$ by $\Theta$, i.e. $\Theta=\{u \in$ $\left.C^{2}(\Omega) \mid\left(W_{i j}\left(D^{2} u\right)(x)\right) \in \Gamma_{2}, x \in \Omega\right\}$.

Remark 1.1. (1) If $u$ is a $2-$ admissible function, then $F\left(D^{2} u\right)=\sigma_{2}\left(W_{i j}\left(D^{2} u\right)\right)$ is elliptic in $u$. In fact, by a straightforward calculation, we know $F\left(D^{2} u\right)=\sigma_{1}\left(D^{2} u\right)^{2}+\sigma_{2}\left(D^{2} u\right)$. So, by the chain rule, we have

$$
\left(\frac{\partial F}{\partial u_{i j}}\right)=\left[\begin{array}{ccc}
\frac{\partial \sigma_{2}}{\partial W_{11}}+\frac{\partial \sigma_{2}}{\partial W_{22}} & \frac{\partial \sigma_{2}}{\partial W_{32}} & -\frac{\partial \sigma_{2}}{\partial W_{31}} \\
\frac{\partial \sigma_{2}}{\partial W_{23}} & \frac{\partial \sigma_{2}}{\partial W_{11}}+\frac{\partial \sigma_{2}}{\partial W_{33}} & \frac{\partial \sigma_{2}}{\partial W_{21}} \\
-\frac{\partial \sigma_{2}}{\partial W_{13}} & \frac{\partial \sigma_{2}}{\partial W_{12}} & \frac{\partial \sigma_{2}}{\partial W_{22}}+\frac{\partial \sigma_{2}}{\partial W_{33}}
\end{array}\right] .
$$

If we denote $\lambda_{i}^{\prime}$ the eigenvalues of $\left(\frac{\partial \sigma_{2}}{\partial W_{i j}}\right),(i=1,2,3)$, then by an orthogonal transformation, we know $\lambda_{1}^{\prime}+\lambda_{2}^{\prime}, \lambda_{1}^{\prime}+\lambda_{3}^{\prime}, \lambda_{2}^{\prime}+\lambda_{3}^{\prime}$ are the eigenvalues of $\left(\frac{\partial F}{\partial u_{i j}}\right)$. Since $u$ is $2-$ admissible, $\left(W_{i j}\left(D^{2} u\right)\right) \in \Gamma_{2}$, we have $\left(\frac{\partial \sigma_{2}}{\partial W_{i j}}\right)>0$ in $\Omega$, i.e. $\lambda_{i}^{\prime}>0$, so are $\lambda_{1}^{\prime}+\lambda_{2}^{\prime}, \lambda_{1}^{\prime}+\lambda_{3}^{\prime}, \lambda_{2}^{\prime}+\lambda_{3}^{\prime}$, that is $\left(\frac{\partial F}{\partial u_{i j}}\right)>0$ in $\Omega$.

(2) If $u$ is a $2-a d m i s s i b l e$ function, then $G\left(D^{2} u\right)=F^{\frac{1}{2}}\left(D^{2} u\right)$ is concave with respect to $D^{2} u$. In fact, we know $\sigma_{2}^{\frac{1}{2}}\left(W_{i j}\right)$ is concave with respect to $\left(W_{i j}\right)$ because $\left(W_{i j}\left(D^{2} u\right)\right) \in \Gamma_{2}$. Since $W_{i j}\left(D^{2} u\right)$ is linear in $u_{i j}$, we know $G\left(D^{2} u\right)$ is concave with respect to $u_{i j}$.

In the following, we will call a 2-admissible function an admissible one for short. Moreover, a solution to (1.1) is called an admissible solution if it is admissible in $\Omega$.

Now, we can state our results. Our main purpose here is to derive some convexity for the solution of (1.1). But before doing that, we need first get the existence and uniqueness result for (1.1).

Theorem 1.1. Let $\Omega \subset R^{3}$ be a bounded domain in $R^{3}$ with smooth boundary. Then, there exists a positive constant $\lambda_{1}$, which depends only on $\Omega$, so that

(1) (1.1) possesses a negative admissible solution $\psi \in C^{\infty}(\Omega) \cap C^{1,1}(\bar{\Omega})$ for $\lambda=\lambda_{1}$,

(2) if $\left(\lambda^{\star}, \psi^{\star}\right) \in[0,+\infty) \times\left(C^{\infty}(\Omega) \cap C^{1,1}(\bar{\Omega})\right)$ is another solution of $(1.1)$, then $\lambda^{\star}=\lambda_{1}, \psi^{\star}=$ $\alpha \psi_{1}$ for some positive constant $\alpha$,

(3) if $\Omega_{1} \subset \Omega_{2}$, then $\lambda_{1}\left(\Omega_{1}\right) \geq \lambda_{1}\left(\Omega_{2}\right)$.

Then, we get the convexity result for (1.1). 
Theorem 1.2. Let $\Omega \subset R^{3}$ be a strictly convex bounded domain in $R^{3}$ with smooth boundary. Let $u \in C^{\infty}(\Omega) \cap C^{1,1}(\bar{\Omega})$ be an admissible solution of $(1.1)$, then $-\log (-u)$ is strictly convex in $\Omega$.

The plan of the paper is as follows. In Section 2, we derive a prior estimate for a general class of equations which will be used in the proof of Theorem 1.1. We finish the proof of Theorem 1.1 in Section 3. Then, we proved the constant rank theorem in Section 4 , which is essential to derive the convexity results. In Section 5, we use the deformation technique together with the constant rank theorem to get the proof of Theorem 1.2.

\section{$2 C^{1}$ and $C^{2}$ estimates}

Firstly, we derive the existence and uniqueness result for (1.1). Our method is similar to that of Wang [33]. To do so, we need first get a prior estimate for the following Dirichlet problem.

Consider the Dirichlet problem

$$
\begin{cases}F\left(D^{2} u\right)=f(x, u) & \text { in } \Omega, \\ u=\varphi & \text { on } \partial \Omega\end{cases}
$$

where $F\left(D^{2} u\right)=\sigma_{2}\left(W_{i j}\left(D^{2} u\right)\right)=\sigma_{1}^{2}\left(D^{2} u\right)+\sigma_{2}\left(D^{2} u\right),\left(W_{i j}\left(D^{2} u\right)\right)$ is defined as in (1.2). Suppose that $f(x, u) \in C^{1,1}(\bar{\Omega} \times R), f \geq 0$, and $\varphi \in C^{3,1}(\partial \Omega)$, we first derive the following a prior $C^{1}$ estimate for the solution of (2.1).

Theorem 2.1. Let $\Omega$ be a bounded domain in $R^{3}$ with smooth boundary. If $u \in C^{3}(\Omega) \cap C^{1}(\bar{\Omega})$ is an admissible solution of (2.1), then

$$
\underset{\Omega}{\sup }|D u| \leq M_{1}
$$

where $M_{1}$ depends only on $\Omega,\|\varphi\|_{C^{3}(\partial \Omega)},|f|_{C^{0}},\left|D_{x} f\right|_{C^{0},}\left|D_{u} f\right|_{C^{0}}$ and $M_{0}=\sup _{\bar{\Omega}}|u|$, where the $C^{0}$ norm of $f, D_{x} f, D_{u} f$ are taken over $\bar{\Omega} \times\left[-M_{0}, M_{0}\right]$.

Proof. We first extend $\varphi$ harmonically into $\Omega$, which is denoted by $\widetilde{\varphi}$. Since $u$ is admissible, $\left(W_{i j}\right)(x) \in \Gamma_{2}, x \in \Omega$, we have $\triangle u=\frac{1}{2} \sigma_{1}\left(W_{i j}\left(D^{2} u\right)\right)>0$ in $\Omega$, i.e. $u$ is subharmonic in $\Omega$. So, from the comparison principle, we know

$$
u \leq \widetilde{\varphi} \quad \text { in } \Omega .
$$

Then, for any point $x_{0} \in \partial \Omega$, we may assume that $x_{0}$ is the origin and the positive $x_{3}$ axis is the interior normal there and $x_{i}(1 \leq i \leq 2)$ are the principal directions of $\partial \Omega$ at $x_{0}$. So, $\partial \Omega$ can locally be represented as

$$
x_{3}=\rho\left(x^{\prime}\right)=\frac{1}{2} \sum_{1 \leq i \leq 2} \kappa_{i} x_{i}^{2}+O\left(\left|x^{\prime}\right|^{3}\right),
$$


where $\kappa_{i}(1 \leq i \leq 2)$ are the principal curvatures of $\partial \Omega$ at $x_{0}, x^{\prime}=\left(x_{1}, x_{2}\right)$. Near $\partial \Omega$, let $d(x)$ denote the distance function to $\partial \Omega$. At the origin we have $\left(d_{i j}\right)=\operatorname{diag}\left(-\kappa_{1},-\kappa_{2}, 0\right)$. For large $t$, we consider $v=\frac{1}{t}\left(e^{-t d}-1\right)$, then at the origin

$$
\left(v_{i j}\right)=\left(-d_{i j}+t d_{i} d_{j}\right)=\operatorname{diag}\left(\kappa_{1}, \kappa_{2}, t\right) .
$$

From the definition of $\left(W_{i j}\left(D^{2} v\right)\right)$, we know

$$
\left(W_{i j}\left(D^{2} v\right)\right)=\operatorname{diag}\left(t+\kappa_{1}, t+\kappa_{2}, \kappa_{1}+\kappa_{2}\right) .
$$

So $\left(W_{i j}\left(D^{2} v\right)\right) \in \Gamma_{2}$ for $t$ large enough. Following the arguments in Caffarelli-NirenbergSpruck [7], we can get a sub-solution $\underline{u}$, such that $\underline{u}$ is admissible in $\bar{\Omega}$ and

$$
\begin{cases}F\left(D^{2} \underline{u}\right) \geq \sup _{(x, u) \in \bar{\Omega} \times\left[-M_{0}, M_{0}\right]} f(x, u) & \text { in } \Omega, \\ \underline{u}=\varphi & \text { on } \partial \Omega .\end{cases}
$$

Since both $\underline{u}$ and $u$ are admissible in $\Omega, F$ is elliptic. So, we can use the comparison principle to deduce that

$$
\underline{u} \leq u \quad \text { in } \Omega \text {. }
$$

Combining (2.3) and (2.8) and the fact that $\underline{u}=u=\widetilde{\varphi}=\varphi$ on $\partial \Omega$, we get that

$$
|D u| \leq C \quad \text { on } \partial \Omega \text {. }
$$

Next, we deduce the global gradient estimate.

Let $G(x, \xi)=q(u) u_{\xi}$, where $q(u)=\left(2 M_{0}+1-u\right)^{-\frac{1}{2}}, u_{\xi}$ denotes differentiation in the direction $\xi$. Suppose $G(x, \xi)$ attains its maximum in $\bar{\Omega}$ at $x_{1}$ with $\xi=e_{1}$. If $x_{1} \in \partial \Omega$, then by (2.9), we have done. If $x_{1} \in \Omega$, we have $u_{1}\left(x_{1}\right)=|\nabla u|\left(x_{1}\right)$ and $u_{i}\left(x_{1}\right)=0$ for $2 \leq i \leq 3$. We may assume $u_{1}\left(x_{1}\right)>0$, or there is nothing to prove. All the following calculations are carried out at $x_{1}$. So, we have

$$
\begin{aligned}
& 0=(\log G)_{i}=\frac{q^{\prime}}{q} u_{i}+\frac{u_{1 i}}{u_{1}}, \quad i=1,2,3 \\
& (\log G)_{i j}=\frac{q^{\prime \prime}}{q} u_{i} u_{j}+\frac{q^{\prime}}{q} u_{i j}-\frac{\left(q^{\prime}\right)^{2}}{q^{2}} u_{i} u_{j}+\frac{u_{1 i j}}{u_{1}}-\frac{u_{1 i} u_{1 j}}{u_{1}^{2}}, \quad i, j=1,2,3 .
\end{aligned}
$$

From (2.10), we know

$$
u_{11}=-\frac{q^{\prime}}{q} u_{1}^{2} \text { and } u_{1 i}=0, \quad i=2,3
$$

Rotate the $x_{2}, x_{3}$ axis such that $\left\{u_{i j}\right\}_{2 \leq i, j \leq 3}$ is diagonal at $x_{1}$. Then we know $\left\{u_{i j}\right\}_{1 \leq i, j \leq 3}$ and $\left(\frac{\partial F}{\partial u_{i j}}\right)$ are both diagonal at $x_{1}$. From Remark 1.1 , we know $\left(F^{i j}\right):=\left(\frac{\partial F}{\partial u_{i j}}\right)$ is positive 
definite in $\Omega$. Thus

$$
\begin{aligned}
0 & \geq F^{i i}(\log G) i i \\
& =\left[\frac{q^{\prime \prime}}{q}-\left(\frac{q^{\prime}}{q}\right)^{2}\right] F^{i i} u_{i}^{2}+\frac{q^{\prime}}{q} F^{i i} u_{i i}+\frac{F^{i i} u_{i i 1}}{u_{1}}-\frac{F^{i i} u_{1 i}^{2}}{u_{1}^{2}} \\
& =\left[\frac{q^{\prime \prime}}{q}-\left(\frac{q^{\prime}}{q}\right)^{2}\right] F^{i i} u_{i}^{2}+\frac{q^{\prime}}{q} 2 F+\frac{f(x, u)_{1}}{u_{1}}-F^{11}\left(\frac{q^{\prime 2}}{q}\right) \frac{u_{1}^{4}}{u_{1}^{2}} \\
& \geq\left[\frac{q^{\prime \prime}}{q}-2\left(\frac{q^{\prime}}{q}\right)^{2}\right] F^{11} u_{1}^{2}+\frac{q^{\prime}}{q} 2 f+\frac{f_{1}}{u_{1}}+f_{u},
\end{aligned}
$$

where we have used the fact that $F\left(D^{2} u\right)$ is homogeneous of degree 2 (so we have $F^{i j} u_{i j}=$ $2 F)$. Since

$$
\left[\frac{q^{\prime \prime}}{q}-2\left(\frac{q^{\prime}}{q}\right)^{2}\right] \geq \frac{1}{36\left(M_{0}+1\right)^{2}}
$$

we only need to estimate $F^{11}$ to get an upper bound for $u_{1}\left(x_{1}\right)$. In fact, since

$$
F^{11}=\frac{\partial \sigma_{2}}{\partial u_{11}}+2 \sigma_{1}=u_{22}+u_{33}+2 \sigma_{1}
$$

we have

$$
\begin{aligned}
0 & \leq f(x, u)=F\left(D^{2} u\right)=\sigma_{2}\left(D^{2} u\right)+\sigma_{1}^{2}\left(D^{2} u\right) \\
& =F^{11} u_{11}+\sigma_{2}\left(D^{2} u \mid 1\right)+\sigma_{1}^{2}\left(D^{2} u\right)-2 \sigma_{1} u_{11} \\
& =F^{11} u_{11}+u_{22} u_{33}+\sigma_{1}\left(u_{22}+u_{33}-u_{11}\right) \\
& \leq F^{11} u_{11}+\frac{\left(u_{22}+u_{33}\right)^{2}}{4}+2 \sigma_{1}\left(u_{22}+u_{33}\right) \\
& \leq F^{11} u_{11}+\frac{1}{2}\left(F^{11}\right)^{2}=-F^{11} \frac{q^{\prime}}{q} u_{1}^{2}+\frac{1}{2}\left(F^{11}\right)^{2},
\end{aligned}
$$

where we use the cauchy inequality and the fact that $\Delta u>0$, i.e. $-u_{11}<u_{22}+u_{33}$ for the second " $\leq$ " and we use (2.15) for the last one. It then follows from (2.16) that (notice that $F^{11}>0$ since $\left(F^{i j}\right)>0$ )

$$
F^{11} \geq 2 \frac{q^{\prime}}{q} u_{1}^{2} \geq \frac{1}{6\left(M_{0}+1\right)} u_{1}^{2}
$$

Inserting (2.14) and (2.17) into (2.13), we get an upper bound for $u_{1}\left(x_{1}\right)=|\nabla u|\left(x_{1}\right)$, which also implies a global upper bound for $|\nabla u|$.

Now, we derive the a prior $C^{2}$ estimate of $(2.1)$ when $\varphi \equiv 0$ on $\partial \Omega$. 
Theorem 2.2. Let $\Omega$ be a bounded domain in $R^{3}$ with smooth boundary. Suppose $\varphi=0$ on $\partial \Omega$ and

$$
\begin{aligned}
& D_{x}\left(f^{\frac{1}{2}}\right), D_{u}\left(f^{\frac{1}{2}}\right) \in C^{1}(\bar{\Omega} \times R), \\
& \left|D_{x}\left(f^{\frac{1}{2}}\right)\right|_{C^{0}},\left|D_{u}\left(f^{\frac{1}{2}}\right)\right|_{C^{0}} \leq C_{1}<+\infty, \\
& \left|D_{x x}^{2}\left(f^{\frac{1}{2}}\right)\right|_{C^{0}},\left|D_{x u}^{2}\left(f^{\frac{1}{2}}\right)\right|_{C^{0}},\left|D_{u u}^{2}\left(f^{\frac{1}{2}}\right)\right|_{C^{0}} \leq C_{2}<+\infty .
\end{aligned}
$$

Then if $u \in C^{3,1}(\Omega) \cap C^{3}(\bar{\Omega})$ is an admissible solution of $(2.1)$, we have

$$
\underset{\Omega}{\sup }\left|D^{2} u\right| \leq M_{2}
$$

where $M_{2}$ depends only on $\Omega,\|f\|_{C^{0}}, C_{1}, C_{2},\|\nabla u\|_{C^{0}(\bar{\Omega})}$, where the $C^{0}$ norms related to $f$ are taken over $\bar{\Omega} \times\left[-M_{0}, M_{0}\right]$.

Proof. The outline of our proof is similar to that of Theorem 3.2 in [33]. Rewrite the equation in (2.1) as

$$
G\left(D^{2} u\right)=\sigma_{2}^{\frac{1}{2}}\left(W_{i j}\left(D^{2} u\right)\right)=g(x, u) \quad \text { in } \Omega,
$$

where $g=f^{\frac{1}{2}}$, and denote

$$
G_{i j}(r)=\frac{\partial G}{\partial r_{i j}}(r), \quad G_{i j, s t}(r)=\frac{\partial^{2} G}{\partial r_{s t} \partial r_{i j}}(r),
$$

then we have

$$
\begin{aligned}
& G_{i j} D_{i j} u_{k}=D_{k} g, \\
& G_{i j} D_{i j} u_{k l}=-G_{i j, s t} u_{i j k} u_{s t l}+D_{k l} g .
\end{aligned}
$$

Since $u$ is admissible, $G\left(D^{2} u\right)$ is concave with respect to $u_{i j}$ by Remark 1.1 (2). So from (2.22) and (2.18), we know

$$
G_{i j} D_{i j}(\triangle u) \geq \triangle g \geq g_{u} \triangle u-C
$$

Observing that

$$
G_{i j}\left(D^{2} u\right)=\frac{1}{2} \sigma_{2}^{-\frac{1}{2}}\left(W_{i j}\right) \frac{\partial \sigma_{2}\left(W_{i j}\right)}{\partial u_{i j}}
$$

From Remark 1.1 (1), we know that if we denote $\lambda_{i}^{\prime}$ the eigenvalues of $\left(\frac{\partial \sigma_{2}\left(W_{i j}\right)}{\partial W_{i j}}\right)(i=1,2,3)$, then $\lambda_{1}^{\prime}+\lambda_{2}^{\prime}, \lambda_{1}^{\prime}+\lambda_{3}^{\prime}, \lambda_{2}^{\prime}+\lambda_{3}^{\prime}$ are the eigenvalues of $\left(\frac{\partial \sigma_{2}\left(W_{i j}\right)}{\partial u_{i j}}\right)$ and all positive. So, by the 
Newton-MacLaurin inequality, we have

$$
\begin{aligned}
\operatorname{det}\left(\frac{\partial \sigma_{2}\left(W_{i j}\right)}{\partial u_{i j}}\right) & =\left(\lambda_{1}^{\prime}+\lambda_{2}^{\prime}\right)\left(\lambda_{1}^{\prime}+\lambda_{3}^{\prime}\right)\left(\lambda_{2}^{\prime}+\lambda_{3}^{\prime}\right)=\sigma_{1}\left(\lambda^{\prime}\right) \sigma_{2}\left(\lambda^{\prime}\right)-\sigma_{3}\left(\lambda^{\prime}\right) \\
& \geq 8 \sigma_{3}\left(\lambda^{\prime}\right)=8 \operatorname{det}\left(\frac{\partial \sigma_{2}\left(W_{i j}\right)}{\partial W_{i j}}\right) \geq 8 C\left[\sigma_{2}\left(W_{i j}\right)\right]^{\frac{3}{2}}
\end{aligned}
$$

where $C=\left(\frac{2}{3}\right)^{3} 3^{\frac{3}{2}}$ (see Prop 2.2 in [33]). So we have $\operatorname{det}\left(G_{i j}\right) \geq C$. Applying the Alexandrov maximum principle to (2.23), we obtain (since $\triangle u \geq 0$ )

$$
\begin{aligned}
\sup _{\Omega} \Delta u & \leq \sup _{\partial \Omega} \Delta u+C\|1+\Delta u\|_{L^{3}(\Omega)} \\
& \leq \sup _{\partial \Omega} \Delta u+C \sup _{\Omega}(1+\Delta u)^{\frac{2}{3}}\left(\int_{\Omega}(1+\triangle u) d x\right)^{\frac{1}{3}} \\
& \leq \sup _{\partial \Omega} \Delta u+C \sup _{\Omega}(1+\triangle u)^{\frac{2}{3}}
\end{aligned}
$$

where we use the divergence theorem and the $C^{1}$ estimate in (2.2) for the last " $\leq$ ". So, we have

$$
\sup _{\Omega} \Delta u \leq C\left(1+\sup _{\partial \Omega} \triangle u\right) .
$$

For any fixed $x \in \Omega$, by an orthogonal transformation of coordinates we may suppose $\left(u_{i j}\right)(x)$ is diagonal, then $\left(W_{i j}\left(D^{2} u\right)(x)\right)$ is also diagonal. Since $\left(W_{i j}\right)$ is in $\Gamma_{2}$, we have $W_{i i}+W_{j j}>0, i, j=1,2,3, i \neq j$, i.e.

$$
u_{i i}>-\triangle u, \quad i=1,2,3 \text {. }
$$

On the other hand, we have

$$
W_{i i}+W_{j j} \leq\left(W_{11}+W_{22}\right)+\left(W_{11}+W_{33}\right)+\left(W_{22}+W_{33}\right)=4 \triangle u, \quad i, j=1,2,3, i \neq j,
$$

i.e. $\triangle u+u_{i i} \leq 4 \triangle u, i=1,2,3$ or

$$
u_{i i} \leq 3 \triangle u, \quad i=1,2,3 .
$$

In other words, we have $\left|u_{i i}\right| \leq 3 \triangle u, i=1,2,3$. Since $i$ is arbitrary and $\Delta u$ is invariant under rotation, we can rotate the coordinates to get

$$
\left|D^{2} u\right| \leq C|\triangle u|=C \triangle u \quad \text { in } \Omega .
$$

Combining (2.28) and (2.25), we get

$$
\sup _{\Omega}\left|D^{2} u\right| \leq C\left(1+\sup _{\partial \Omega}\left|D^{2} u\right|\right) .
$$


It remains to estimate $\left|D^{2} u\right|$ on the boundary. For any given $x \in \partial \Omega$, we may suppose it is the origin and $\partial \Omega$ can locally be represented as in (2.4) near the origin. Since $u=\varphi=0$ on $\partial \Omega$, we have

$$
u_{\alpha \beta}(0)=-u_{3} \kappa_{\alpha} \delta_{\alpha \beta}, \quad 1 \leq \alpha, \beta \leq 2 .
$$

Let $\widetilde{w}=\rho\left(x^{\prime}\right)-x_{3}-\delta\left|x^{\prime}\right|^{2}+K x_{3}^{2}$, then it is easy to check that $\widetilde{w}$ is admissible in a small neighborhood of $x$ in $\Omega$ since $\left(W_{i j}\left(D^{2} \widetilde{w}\right)\right)$ has eigenvalues $\kappa_{1}+\kappa_{2}-4 \delta, 2 K+\kappa_{1}-2 \delta, 2 K+$ $\kappa_{2}-2 \delta$ at $x$. So $G\left(D^{2} \widetilde{w}\right)$ is concave with respect to $D^{2} \widetilde{w}$, we can use the similar arguments as in Caffarelli-Nirenberg-Spruck [7] or Wang [34] to show that

$$
\left|u_{\alpha 3}(0)\right| \leq C, \quad 1 \leq \alpha \leq 2 .
$$

To estimate $\left|u_{33}(0)\right|$, we first note that $u$ is subharmonic in $\Omega$ (and in $\bar{\Omega}$ by continuity). This together with (2.30) implies a lower bound for $u_{33}(0)$. On the other hand, by (2.30), we have

$$
\left(W_{i j}\left(D^{2} u\right)(0)\right)=\left[\begin{array}{ccc}
-u_{3}\left(\kappa_{1}+\kappa_{2}\right) & u_{32} & -u_{31} \\
u_{23} & -u_{3} \kappa_{1}+u_{33} & 0 \\
-u_{13} & 0 & -u_{3} \kappa_{2}+u_{33}
\end{array}\right] .
$$

So we have

$$
\begin{aligned}
C \geq f(x, 0) & =\sigma_{2}\left(W_{i j}\left(D^{2} u\right)(0)\right) \\
& =u_{33}^{2}-3 u_{33} u_{3}\left(\kappa_{1}+\kappa_{2}\right)+u_{3}^{2}\left(\kappa_{1}{ }^{2}+\kappa_{2}{ }^{2}+3 \kappa_{1} \kappa_{2}\right)-u_{13}^{2}-u_{23}^{2} .
\end{aligned}
$$

Since $u_{3}, u_{13}, u_{23}$ are all bounded, this implies that $u_{33}(0) \leq C$.

Remark 2.1. (1) If $f \in C^{1,1}(\bar{\Omega} \times R)$ is strictly positive in $\bar{\Omega}$, then by Theorem $2.1,2.2$ and the standard elliptic regularity theory we have

$$
\|u\|_{C^{2, \alpha}(\bar{\Omega})} \leq C .
$$

(2) If $f$ satisfies (2.18) and is strictly positive in $\Omega$, then by the interior Hölder estimates for second order derivatives, we have $\|u\|_{C^{2, \alpha}\left(\bar{\Omega}_{1}\right)} \leq C$ for any $\Omega_{1} \subset \subset \Omega$. Hence $u \in C^{2, \alpha}(\Omega) \cap C^{1,1}(\bar{\Omega})$.

(3) Thanks to the special structure of $\left(W_{i j}\left(D^{2} u\right)\right)$ with respect to $D^{2} u$ in the case $k=2$, $n=3$ (i.e. the eigenvalues of $\left(W_{i j}\left(D^{2} u\right)\right)$ are the "choosing sum" of that of $\left.D^{2} u\right)$, we did not make any assumptions on the convexity of $\partial \Omega$ to get the boundary $C^{1}$ and $C^{2}$ estimates here. However, for general $k$ and $n$, we may need $\partial \Omega$ to satisfy some convexity condition which was studied in Harvey-Lawson [18-20] to get these estimates.

From Theorem 2.1 and 2.2 and Remark 2.1.(2), we therefore obtain the existence result for the homogenous Dirichlet problem by the method of super and sub solution. 
Theorem 2.3. Suppose $f \in C^{1,1}(\bar{\Omega} \times R)$ satisfies (2.18) and

$$
f(x, u)>0 \quad \text { for } u<0 .
$$

If there are a sub-solution $w$ and a super-solution $v$ to

$$
\begin{cases}F\left(D^{2} u\right)=f(x, u) & \text { in } \Omega, \\ u=0 & \text { on } \partial \Omega .\end{cases}
$$

then (2.36) possesses a solution $u \in C^{2, \alpha}(\Omega) \cap C^{1,1}(\bar{\Omega})$ with $v \geq u \geq w$.

A function is said to be a sub-solution (resp. super-solution) of (2.36), if

$$
\begin{cases}F\left(D^{2} u\right) \geq(\text { resp. } \leq) f(x, u) & \text { in } \Omega \\ u=0 & \text { on } \partial \Omega .\end{cases}
$$

Proof. This can be derived from the uniform $C^{2, \alpha}$ estimate in Remark 2.1 and the standard super-sub solution method (see Wang [32] or Aubin [2]).

\section{Proof of Theorem 1.1}

In this section, we will use the results in Section 2 to prove Theorem 1.1. First, let's state a lemma which will be used in the proof. This lemma was got by Geng-Yu-Qu [12].

Lemma 3.1. Suppose $u \in C^{2}(\bar{\Omega})$ and $\triangle u>0$ in $\Omega$. If there exists $x_{0} \in \Omega$, positive constants $R$ and $\delta$ such that

$$
\triangle u \geq \delta, \quad x \in B_{R}\left(x_{0}\right) \subset \Omega,
$$

then

$$
\frac{\partial u}{\partial v}(x) \geq \alpha, \quad x \in \partial \Omega,
$$

where $v$ is the unit outer normal of $\partial \Omega, \alpha>0$ depends only on $\Omega, R, \delta$ and $x_{0}$ 's position in $\Omega$.

We now prove Theorem 1.1. The proof is similar to that in $[12,25,33]$.

Proof. (1) Let's first introduce a set $\Lambda$ and a nonnegative constant $\lambda_{1}$ by

$$
\begin{gathered}
\Lambda=\left\{\lambda \geq 0 \mid \text { there is a solution } u_{\lambda} \in C^{2}(\bar{\Omega}) \text { of }(3.4)\right\} \\
\lambda_{1}=\sup \Lambda,
\end{gathered}
$$

where (3.4) is defined as

$$
\begin{cases}F\left(D^{2} u\right)=(1-\lambda u)^{2} & \text { in } \Omega, \\ u=0 & \text { on } \partial \Omega .\end{cases}
$$


and $F\left(D^{2} u\right)=\sigma_{2}\left(W_{i j}\left(D^{2} u\right)\right)$ is the same as in Section 2. We will show that $\lambda_{1}$ is positive and finite, and that as $\lambda \rightarrow \lambda_{1}, \frac{u_{\lambda}}{\left\|u_{\lambda}\right\|_{C^{0}}}$ converges to a solution of (1.1) with $\lambda=\lambda_{1}$ there.

We know from Remark 2.1 (3) and Caffarelli-Nirenberg-Spruck [7] that

$$
\begin{cases}F\left(D^{2} u\right)=1 & \text { in } \Omega, \\ u=0 & \text { on } \partial \Omega .\end{cases}
$$

admits an admissible solution $\eta$. Then,

$$
F\left(D^{2}(2 \eta)\right)=2^{2} \geq(1-2 \lambda \eta)^{2} \text { for } \lambda \in\left(0, \frac{1}{2 \sup _{\Omega}|\eta|}\right) .
$$

From Theorem 2.3 and Remark 2.1, we obtain a solution $u \in C^{2, \alpha}(\bar{\Omega})$ of (3.4) for $\lambda \in$ $\left(0, \frac{1}{2 \sup |\eta|}\right)$. Hence $\lambda_{1}>0$.

To see that $\lambda_{1}$ is finite, just observe that if $(\lambda, u)$ is a solution of (3.4), then we have $\left(W_{i j}\left(D^{2} u\right)\right) \in \bar{\Gamma}_{2}$. So $u$ is subharmonic in $\Omega$ and $u \leq 0$ in $\Omega$. So, by (3.4), we have $\sigma_{2}\left(W_{i j}\left(D^{2} u\right)\right)>0$ in $\Omega$ and by the Newton-Macularin inequality, we have $\Delta u>0$ in $\Omega$, which implies that $u<0$ in $\Omega$. By Newton-Macularin inequality, we have

$$
\triangle u=\frac{1}{2} \sigma_{1}\left(W_{i j}\left(D^{2} u\right)\right) \geq C \sigma_{2}^{\frac{1}{2}}\left(W_{i j}\left(D^{2} u\right)\right)=C(1-\lambda u) .
$$

Let $\bar{\lambda}$ be the first eigenvalue of $-\frac{\Delta}{C}$ with homogenous Dirichlet boundary value and $\psi$ be a positive eigenfunction corresponding to $\bar{\lambda}$. Multiplying (3.6) by $\psi$ and integrating by parts, we have

$$
\int_{\Omega}(\lambda u-1) \psi d x \geq \int_{\Omega}-\frac{\triangle u}{C} \psi d x=\int_{\Omega}-\frac{\triangle \psi}{C} u d x=\bar{\lambda} \int_{\Omega} \psi u d x
$$

i.e.

$$
(\bar{\lambda}-\lambda) \int_{\Omega} \psi u d x \leq-\int_{\Omega} \psi d x<0
$$

Since $\psi u<0$ in $\Omega$, this implies that $\lambda_{1} \leq \bar{\lambda}<+\infty$.

From the definition of $\lambda_{1}$, we see that for any $\lambda_{0} \in\left[0, \lambda_{1}\right)$, there exists $\lambda_{2}, \lambda_{0} \leq \lambda_{2}<\lambda_{1}$ s.t. $\lambda_{2} \in \Lambda$. So $u_{\lambda_{2}}$ is a sub-solution of (3.4) for $\lambda=\lambda_{0}$. By Theorem 2.3, we know $\lambda_{0} \in \Lambda$, i.e. $\left[0, \lambda_{1}\right) \subset \Lambda$. Next, we claim $\left\|u_{\lambda}\right\|_{C^{0}}$ tends to $+\infty$ as $\lambda \rightarrow \lambda_{1}$. In deed, if it is not the case, then there exists a sequence $\left\{\gamma_{i}\right\}_{i=1}^{+\infty} \subset \Lambda$, s.t $\gamma_{i} \rightarrow \lambda_{1}$ with $\left\|u_{\gamma_{i}}\right\|_{C^{0}} \leq C$ where $C$ is independent of $i$. By Theorem 2.1 and Theorem 2.2, we know $\left\|u_{\gamma_{i}}\right\|_{C^{2}(\overline{\Omega)}} \leq C$ with $C$ independent of $i$. Since $f\left(x, u_{\gamma_{i}}\right) \geq 1$ for $x \in \bar{\Omega}$, by Remark 2.1, we know $\left\|u_{\gamma_{i}}\right\|_{C^{2, \alpha}(\bar{\Omega})} \leq C$ with $C$ independent of $i$. So there is a subsequence of $\left\{u_{\gamma_{i}}\right\}$ that converges to some function $u^{\star}$ in $C^{2}(\bar{\Omega})$. Clearly, $\left(\lambda_{1}, u^{\star}\right)$ is a solution of (3.4) and $u^{\star} \not \equiv 0$. Take $\delta>0$, such that $\delta<1 /\left\|u^{\star}\right\|_{C^{0}}$ and set $\underline{u}=\left(1-\delta\left\|u^{\star}\right\|_{C^{0}}\right)^{-1} u^{\star}$, then $\underline{u}=0$ on $\partial \Omega$ and since

$$
\frac{1-\lambda_{1} u^{\star}}{1-\delta\left\|u^{\star}\right\|_{C^{0}}} \geq 1-\left(\lambda_{1}+\delta\right) \underline{u}
$$


we have

$$
F\left(D^{2} \underline{u}\right)=\left(1-\delta\left\|u^{\star}\right\|_{C^{0}}\right)^{-2}\left(1-\lambda_{1} u^{\star}\right)^{2} \geq\left(1-\left(\lambda_{1}+\delta\right) \underline{u}\right)^{2} .
$$

Hence by Theorem 2.3, we have $\lambda_{1}+\delta \in \Lambda$. This contradicts to the definition of $\lambda_{1}$. So the claim is true.

Denote $v_{\lambda}=u_{\lambda} /\left\|u_{\lambda}\right\|_{C^{0}}$, then $v_{\lambda} \in[-1,0]$ and satisfies

$$
\begin{cases}F\left(D^{2} v\right)=\left(\left\|u_{\lambda}\right\|_{C^{0}}^{-1}-\lambda v\right)^{2} & \text { in } \Omega, \\ v=0 & \text { on } \partial \Omega .\end{cases}
$$

Since $\left\|v_{\lambda}\right\|_{C^{0}}$ is uniformly bounded, by Theorem 2.1 and Theorem 2.2 we have

$$
\left\|v_{\lambda}\right\|_{C^{2}(\bar{\Omega})} \leq C
$$

where $C$ is independent of $\lambda$. Hence there is a subsequence of $\left\{v_{\lambda}\right\}$, we denote it by $v_{\mu_{j}}=v_{j}$, such that $\left(\mu_{j}>\frac{\lambda_{1}}{2}>0\right.$ and $) v_{j}$ tends to some function $v_{0} \in C^{0}(\bar{\Omega})$ uniformly in $\bar{\Omega}$. Since $\left\|v_{j}\right\|_{C^{0}(\bar{\Omega})} \equiv 1$, we have $\left\|v_{0}\right\|_{C^{0}(\bar{\Omega})}=1$. Since $v_{0}=0$ on $\partial \Omega$, there exists $x_{0} \in \Omega, R>0$ and $\delta>0$ such that $v_{0}\left(x_{0}\right)=-1, B_{2 R}\left(x_{0}\right) \subset \Omega$ and

$$
v_{0}(x) \leq-2 \delta, \quad x \in B_{2 R}\left(x_{0}\right) .
$$

Thus there is $N$, such that

$$
v_{j}(x) \leq-\delta, \quad j \geq N, x \in B_{R}\left(x_{0}\right) .
$$

On the other hand, since $v_{j}<0$ in $\Omega$ and $\left(W_{i j}\left(D^{2} v_{j}\right)\right) \in \Gamma_{2}$, we have

$$
\triangle v_{j} \geq C \sigma_{2}^{\frac{1}{2}}\left(W_{i j}\left(D^{2} v_{j}\right)\right) \geq-\mu_{j} v_{j}>0 \text { in } \Omega
$$

and $\triangle v_{j} \geq \frac{\lambda_{1}}{2} \delta$ in $B_{R}\left(x_{0}\right), j \geq N$. Set $B_{R}=B_{R}\left(x_{0}\right), r=\left|x-x_{0}\right|\left(x \in R^{3}\right)$ and let

$$
w_{j}(x)=v_{j}(x)-\varepsilon \varphi(r) v(x), \quad j \geq N, x \in \bar{\Omega},
$$

where $\varphi \in C^{\infty}[0,+\infty)$ satisfying $0 \leq \varphi(r) \leq 1 ;\left|\varphi^{\prime}\right|,\left|\varphi^{\prime \prime}\right| \leq M$ and

$$
\varphi(r)= \begin{cases}0 & r \leq \frac{R}{2}, \\ 1 & r \geq R,\end{cases}
$$

$v$ is the unique solution of

$$
\begin{cases}\triangle u=0 & \text { in } \Omega-\bar{B}_{\frac{R}{2}}, \\ u=-1 & \text { on } \partial B_{\frac{R}{2}}, \\ u=0 & \text { on } \partial \Omega .\end{cases}
$$


Then, similar to the proof of Lemma 3.1 in [12], we have $\triangle w_{j}>0$ in $\Omega$ for $\varepsilon>0$ small enough. So by the maximum principle, $w_{j}<0$ in $\Omega$, hence together with (3.13), we have

$$
v_{j}(x) \leq \begin{cases}\varepsilon v(x), & r=\left|x-x_{0}\right|>R \\ -\delta, & r=\left|x-x_{0}\right| \leq R\end{cases}
$$

Therefore, for any open set $\Omega^{\prime} \subset \subset \Omega$, there is $\sigma=\sigma\left(\Omega^{\prime}\right)>0$ such that

$$
v_{j}(x) \leq-\sigma, \quad j \geq N, x \in \Omega^{\prime} .
$$

So, from Remark 2.1 (2), there is $C=C\left(\sigma, \Omega^{\prime}\right)>0$ such that

$$
\left\|v_{j}\right\|_{C^{2+\alpha}\left(\Omega^{\prime}\right)} \leq C,
$$

where $0<\alpha<1, C$ is independent of $j$. Hence there is a subsequence of $\left\{v_{j}\right\}$ converges to some function $\psi_{1} \in C^{\infty}(\Omega) \cap C^{1,1}(\bar{\Omega})$, which is a solution of $(1.1)$ with $\lambda=\lambda_{1},\left(W_{i j}\left(D^{2} \psi_{1}\right)\right) \in$ $\bar{\Gamma}_{2}$ in $\Omega$. On the other hand, we have $\psi_{1}<0$ in $\Omega$ by (3.18). So by (1.1) we have $\sigma_{2}\left(W_{i j}\left(D^{2} \psi_{1}\right)\right)>0$ in $\Omega$ and together with the Newton-Macularin inequality, we have $\left(W_{i j}\left(D^{2} \psi_{1}\right)\right) \in \Gamma_{2}$ in $\Omega$, i.e. $\psi_{1}$ is admissible.

(2) If $\left(\lambda_{1}, u_{1}\right),\left(\lambda_{2}, u_{2}\right) \in[0,+\infty) \times\left(C^{\infty}(\Omega) \cap C^{1,1}(\bar{\Omega})\right)$ are two pairs solutions of (1.1) with $u_{i}$ admissible in $\Omega, i=1,2$. Let $L_{i}=G^{i j}\left(D^{2} u_{i}\right) \partial_{i j}$, then

$$
L_{i} u_{i}=G\left(D^{2} u_{i}\right)=-\lambda_{i} u_{i} \quad \text { in } \Omega, i=1,2 .
$$

By the properties of the first eigenvalue of non-divergence form elliptic operators (see Evans [9] chapter 6.5 Theorem 3), we have

$$
\lambda_{i}=\operatorname{Re}\left(\lambda_{i}\right) \geq \lambda_{1}\left(-L_{i}\right)=-\lambda_{1}\left(L_{i}\right), \quad i=1,2 .
$$

where $\lambda_{1}\left(L_{i}\right)$ is the first eigenvalue of $L_{i}$.

On the other hand, since $\left(W_{i j}\left(D^{2} u_{i}\right)\right) \in \Gamma_{2}$ in $\Omega$. By the concavity and homogeneity of $G$, we have

$$
L_{1} u_{2} \geq G\left(u_{1}+u_{2}\right)-G\left(u_{1}\right) \geq G\left(u_{1}\right)+G\left(u_{2}\right)-G\left(u_{1}\right)=G\left(u_{2}\right)=-\lambda_{2} u_{2} \quad \text { in } \Omega .
$$

By the characterization of the first eigenvalue in Nirenberg [3], we know

$$
-\lambda_{1}\left(L_{1}\right)=\sup \left\{\lambda \in R \mid \exists \phi>0 \text { in } \Omega \text {, s.t. } L_{1} \phi+\lambda \phi \leq 0 \text { in } \Omega\right\} \text {, }
$$

where $\phi$ is in $W_{l o c}^{2, p}(\Omega)$. Since $-u_{2}>0$ in $\Omega$ and $-u_{2} \in C^{\infty}(\Omega) \subset W_{l o c}^{2, p}(\Omega)$, we have by (3.22) and (3.23) that

$$
\lambda_{2} \leq-\lambda_{1}\left(L_{1}\right),
$$

which together with (3.21) imply that $\lambda_{2} \leq \lambda_{1}$. Similarly, $\lambda_{1} \leq \lambda_{2}$. So $\lambda_{1}=\lambda_{2}$ and all the inequality in (3.21), (3.24) should be" $="$ and $\lambda_{1}=\lambda_{2}=-\lambda_{1}\left(L_{1}\right)=-\lambda_{1}\left(L_{2}\right)$. 
Since $\left(W_{i j}\left(D^{2} u_{2}\right)\right) \in \Gamma_{2}$ in $\Omega$, we have $\Delta u_{2}=\frac{1}{2} \sigma_{1}\left(W_{i j}\left(D^{2} u_{2}\right)\right)>0$ in $\Omega$. By Hopf lemma, we have

$$
\frac{\partial u_{2}}{\partial v}(x)>0, \quad x \in \partial \Omega,
$$

where $v$ is the unit outer normal of $\partial \Omega$. Hence, there exists $t>0$ small such that $t u_{1}-u_{2} \geq 0$ in $\bar{\Omega}$. Let $t_{0}=\sup \left\{t>0 \mid t u_{1}-u_{2} \geq 0\right.$ in $\left.\bar{\Omega}\right\}$, then we have $0<t_{0}<+\infty\left(t_{0}<+\infty\right.$ because $u_{1}<0$ in $\Omega$ ).

By the definition of $t_{0}$, we know $t_{0} u_{1}-u_{2} \geq 0$ in $\bar{\Omega}$. On the other hand, by (3.20) and (3.22), we have

$$
-L_{1}\left(t_{0} u_{1}-u_{2}\right) \geq-t_{0} L_{1} u_{1}-\lambda_{2} u_{2}=\lambda_{1}\left(t_{0} u_{1}-u_{2}\right) \quad \text { in } \Omega \text {. }
$$

If $\exists x_{0} \in \Omega$ such that $\left(t_{0} u_{1}-u_{2}\right)\left(x_{0}\right)=0$, then $t_{0} u_{1}-u_{2}$ attains its minimum at $x_{0} \in$ $\Omega$. Since we have $-L_{1}\left(t_{0} u_{1}-u_{2}\right) \geq 0$ in $\Omega$ by (3.26), So $t_{0} u_{1}-u_{2} \equiv 0$ in $\bar{\Omega}$ by the strong maximum principle and we have done in this case.

If $\left(t_{0} u_{1}-u_{2}\right)(x)>0, \forall x \in \Omega$, then we have $-L_{1}\left(t_{0} u_{1}-u_{2}\right)>0, \forall x \in \Omega$ by (3.26). By Hopf lemma, we have

$$
\frac{\partial\left(t_{0} u_{1}-u_{2}\right)}{\partial v}(x)<0, \quad x \in \partial \Omega
$$

So there exists $\varepsilon>0$ such that $t_{0} u_{1}-u_{2} \geq \varepsilon\left(-u_{1}\right)$ in $\Omega$ (hence in $\bar{\Omega}$ ), i.e. $\left(t_{0}+\varepsilon\right) u_{1}-u_{2} \geq 0$ in $\bar{\Omega}$. This contradicts to the definition of $t_{0}$. So we have $u_{2} \equiv t_{0} u_{1}$ in $\bar{\Omega}$.

(3) Let $\lambda_{i}=\lambda_{1}\left(\Omega_{i}\right)$ with the corresponding admissible eigenfunction $u_{i}, i=1$,2. Since $u_{2}$ is admissible in $\Omega_{2}$, it is also admissible in $\Omega_{1}$. So (3.22) holds in $\Omega_{1}$. By (3.23) and the above argument, we know $\lambda_{2} \leq-\lambda_{1}\left(L_{1}\right)=\lambda_{1}$.

\section{Constant rank theorem}

Let $\Omega \subset R^{3}$ be a strictly convex bounded domain. We now move to the proof of Theorem 1.2. From Theorem 1.1, we know there exists $(\lambda, u) \in(0,+\infty) \times\left(C^{\infty}(\Omega) \cap C^{1,1}(\bar{\Omega})\right)$ be the unique (up to a positive multiplier) admissible solution of (1.1).

As we have known above, $F\left(D^{2} u\right)=\sigma_{2}\left(W_{i j}\left(D^{2} u\right)\right)=\sigma_{1}^{2}\left(D^{2} u\right)+\sigma_{2}\left(D^{2} u\right)$, set $v=-\log (-u)$, then we have

$$
u=-e^{-v}, \quad u_{i}=e^{-v} v_{i}, \quad u_{i j}=e^{-v}\left(v_{i j}-v_{i} v_{j}\right) .
$$

So, (1.1) is equivalent to

$$
\left\{\begin{array}{lr}
\sigma_{2}\left(D^{2} v\right)-\operatorname{tr}\left(M D^{2} v\right)+\left(\sigma_{1}\left(D^{2} v\right)-|D v|^{2}\right)^{2}=\lambda & \text { in } \Omega, \\
v \rightarrow+\infty & \text { on } \partial \Omega .
\end{array}\right.
$$

where $M=M(D v)=|\nabla v|^{2} I-D v D v^{T}$ is a positive semi-definite matrix. To prove the strict convexity of $v$ in $\Omega$, we first derive a constant rank theorem for it. 
Lemma 4.1 (Constant Rank Theorem). Let $\Omega \subset R^{3}$ be a bounded domain, $u \in C^{\infty}(\Omega) \cap C^{1,1}(\bar{\Omega})$ be an admissible solution of (1.1) (so that $v=-\log (-u)$ satisfies (4.2)) and $D^{2} v \geq 0$ in $\Omega$, then $D^{2} v$ is of constant rank in $\Omega$.

Proof. Let $r_{0}$ be the smallest rank of $D^{2} v$ in $\Omega$. We only need to prove $\operatorname{rank}\left(D^{2} v\right) \equiv r_{0}$ in $\Omega$. Firstly, as $u$ is admissible, $\sigma_{1}\left(D^{2} u\right)=\frac{1}{2} \sigma_{1}\left(W_{i j}\left(D^{2} u\right)\right)>0$ in $\Omega$. So $\sigma_{1}\left(D^{2} v\right)=e^{v} \sigma_{1}\left(D^{2} u\right)+$ $|D v|^{2}>0$ by (4.1), $r_{0}$ can only be $1,2,3$. If $r_{0}=3$, then there is nothing to prove. Next, we consider the case when $r_{0}=1,2$.

Set $P=\sigma_{r_{0}+1}\left(D^{2} v\right), \Omega_{1}=\{x \in \Omega \mid P(x)=0\}$. Since $D^{2} v$ is semi-positive in $\Omega$ and $r_{0}$ is the smallest rank of $D^{2} v$ in $\Omega$, we shall prove that

$$
\Omega_{1}=\Omega \text {. }
$$

So there are exactly $r_{0}$ eigenvalues of $\left(D^{2} v\right)^{\prime}$ s which are positive everywhere in $\Omega$, i.e. $\operatorname{rank}\left(D^{2} v\right) \equiv r_{0}$ in $\Omega$. First, we know $\Omega_{1}$ is not empty since $D^{2} v$ must attains the smallest rank $r_{0}$ at some point in $\Omega$. Note that, $\Omega_{1}$ is closed in $\Omega$ from its definition. Now, let $x_{0} \in \Omega_{1}$, we shall prove that there exists a neighborhood $O_{1}$ of $x_{0}$ in $\Omega$ such that $P \equiv 0$ in $O_{1}$, which implies that $\Omega_{1}$ is open. Since $\Omega$ is connected, we must have $\Omega_{1}=\Omega$.

Following the notations in [6] and [23], let $O_{1}$ be any open set and $h, k$ be functions defined in $O_{1}$. Let $y \in O_{1}$, we say $h(y) \precsim k(y)$ if there exist two positive constants $c_{1}, c_{2}$ such that

$$
h(y) \leqslant k(y)+\left(c_{1} P+c_{2}|\nabla P|\right)(y) .
$$

We say $h(y) \sim k(y)$ if both $h(y) \precsim k(y)$ and $k(y) \precsim h(y)$ hold. Next, we say $h \precsim k$ in $O_{1}$ if (4.4) holds in $O_{1}$ with $c_{1}, c_{2}$ independent of $y \in O_{1}$. Finally, we say $h \sim k$ in $O_{1}$ if we both have $h \precsim k$ and $k \precsim h$ hold in $O_{1}$.

Now, let $F=F\left(D v, D^{2} v\right)=\sigma_{2}\left(D^{2} v\right)-\operatorname{tr}\left(M D^{2} v\right)+\left(\sigma_{1}\left(D^{2} v\right)-|D v|^{2}\right)^{2}$, and we use the following notations

$$
F^{i j}=\frac{\partial F}{\partial v_{i j}}, \quad F^{p_{l}}=\frac{\partial F}{\partial v_{l}}, \quad F^{i j, r s}=\frac{\partial^{2} F}{\partial v_{r s} \partial v_{i j}}, \quad F^{i j, p_{l}}=\frac{\partial^{2} F}{\partial v_{l} \partial v_{i j}} .
$$

Fixing a point $x_{0} \in \Omega_{1}$, we shall prove that

$$
F^{i j} P_{i j} \lesssim 0
$$

in an open neighborhood $O_{1} \subset \Omega_{1}$ of $x_{0}$. Then by the strong maximum principle, we know $P \equiv P\left(x_{0}\right)=0$ in $O_{1}$. To prove (4.5), we assume $O_{1} \subset \subset \Omega$. We fix a point $x \in O_{1}$ and rotate the coordinates such that $\left(v_{i j}\right)$ is diagonal and $v_{11} \geq v_{22} \geq v_{33}$ at $x$. We deal with the two cases $\left(r_{0}=1\right.$ and $\left.r_{0}=2\right)$ separately.

Case 1: $r_{0}=2$. In this case, $P(x)=\operatorname{det}\left(D^{2} v(x)\right)$. Since the smallest rank of $D^{2} v$ is 2, there exists a positive constant $c_{1}>0$, depending only on $\|v\|_{C^{3}}$ and $\operatorname{dist}\left(O_{1}, \partial \Omega\right)$, such that $v_{11} \geq v_{22} \geq c_{1}>0$ in $O_{1}$. In the following, we do the calculations at $x$ with the constant 
under control and independent of $x \in O_{1}$. First, since $D^{2} v$ diagonal at $x$ and $v_{11} \geq v_{22} \geq c_{1}>0$ in $O_{1}$, we have $P(x)=v_{11} v_{22} v_{33}$ and $0 \leq v_{33}=\frac{P}{v_{11} v_{22}} \leq \frac{P}{c_{1}^{2}}$, that is

$$
v_{33} \sim P \sim 0
$$

Differentiating $P$ with respect to $x_{i}$ and $x_{j}$, we get

$$
\begin{aligned}
& v_{33 i} \sim P_{i} \sim 0, \\
& P_{i j} \sim v_{11} v_{22} v_{33 i j}-2 v_{22} v_{13 i} v_{13 j}-2 v_{11} v_{23 i} v_{23 j},
\end{aligned}
$$

which implies that

$$
F^{i j} P_{i j} \sim v_{11} v_{22} F^{i j} v_{i j 33}-2 v_{22} F^{i j} v_{13 i} v_{13 j}-2 v_{11} F^{i j} v_{23 i} v_{23 j} .
$$

Now, we compute the partial derivatives of $F$ along $v_{i j}$, it follows that

$$
\begin{aligned}
& F^{11} \sim 2 v_{11}+3 v_{22}-2 v_{1}^{2}-3\left(v_{2}^{2}+v_{3}^{2}\right), \quad F^{22} \sim 2 v_{22}+3 v_{11}-2 v_{2}^{2}-3\left(v_{1}^{2}+v_{3}^{2}\right), \\
& F^{12} \sim v_{2} v_{1} \sim v_{1} v_{2} \sim F^{21}, \quad F^{11,11}=F^{22,22}=2, \\
& F^{11,22}=F^{22,11}=3, \quad F^{12,21}=F^{21,12}=-1 .
\end{aligned}
$$

Next, differentiating (4.2) with respect to $x_{3}$, we have

$$
F^{p_{l}} v_{l 3}+F^{i j} v_{i j 3}=0 .
$$

Differentiating with respect to $x_{3}$ again, we have

$$
F^{i j, r s} v_{i j 3} v_{r s 3}+F^{i j} v_{i j 33} \sim 0 .
$$

By (4.12) and (4.10) we know that

$$
F^{i j} v_{i j 33} \sim-F^{i j, r s} v_{i j 3} v_{r s 3} \sim-2 v_{113}^{2}-2 v_{223}^{2}-6 v_{113} v_{223}+2 v_{123}^{2} .
$$

On the other hand, by (4.11), we have

$$
2 F^{12} v_{123} \sim-F^{11} v_{113}-F^{22} v_{223},
$$

which implies that

$$
F^{i j} v_{13 i} v_{13 j} \sim-F^{22} v_{113} v_{223}+F^{22} v_{123}^{2}, \quad F^{i j} v_{23 i} v_{23 j} \sim-F^{11} v_{113} v_{223}+F^{11} v_{123}^{2} .
$$

Putting (4.13) and (4.15) into (4.9) to substitute corresponding terms, we get

$$
F^{i j} P_{i j} \sim 2\left(v_{11} v_{22}-F^{11} v_{11}-F^{22} v_{22}\right)\left(v_{123}^{2}-v_{113} v_{223}\right)-2 v_{11} v_{22}\left(v_{113}+v_{223}\right)^{2} .
$$


On the other hand, since $v_{33} \sim 0$ and $D^{2} v$ is diagonal at $x$, we have

$$
\begin{aligned}
& \lambda=F\left(D v, D^{2} v\right) \\
\sim & v_{11}^{2}+v_{22}^{2}+3 v_{11} v_{22}-v_{11}\left[2 v_{1}^{2}+3\left(v_{2}^{2}+v_{3}^{2}\right)\right]-v_{22}\left[2 v_{2}^{2}+3\left(v_{1}^{2}+v_{3}^{2}\right)\right]+|D v|^{4} .
\end{aligned}
$$

From (4.10) and (4.17), we know

$$
v_{11} v_{22}-F^{11} v_{11}-F^{22} v_{22} \sim-\sigma_{1}^{2}\left(D^{2} v\right)-\lambda+|D v|^{4} .
$$

Putting (4.18) into (4.16), we have

$$
F^{i j} P_{i j} \sim 2\left(-\sigma_{1}^{2}\left(D^{2} v\right)-\lambda+|D v|^{4}\right)\left(v_{123}^{2}-v_{113} v_{223}\right)-2 v_{11} v_{22}\left(v_{113}+v_{223}\right)^{2} .
$$

Now, we only need to prove $\left(-\sigma_{1}^{2}\left(D^{2} v\right)-\lambda+|D v|^{4}\right)\left(v_{123}^{2}-v_{113} v_{223}\right) \lesssim 0 i n O_{1}$. First, notice that

$$
\sigma_{1}\left(D^{2} v\right)=e^{v} \sigma_{1}\left(D^{2} u\right)+|D v|^{2} \geq|D v|^{2} \geq 0 .
$$

So we have

$$
-\sigma_{1}^{2}\left(D^{2} v\right)-\lambda+|D v|^{4} \leq-\lambda<0 .
$$

Next, we know from (4.17) that

$$
\begin{aligned}
& 4 v_{1}^{2} v_{2}^{2} v_{123}^{2} \\
\succsim & 4\left[2 v_{11}+3 v_{22}-2 v_{1}^{2}-3\left(v_{2}^{2}+v_{3}^{2}\right)\right]\left[2 v_{22}+3 v_{11}-2 v_{2}^{2}-3\left(v_{1}^{2}+v_{3}^{2}\right)\right] v_{113} v_{223} \\
\sim & 4\left[\frac{13}{3} \lambda+\frac{5}{3}\left(v_{11}-v_{1}^{2}-\frac{3}{5} v_{3}^{2}\right)^{2}+\frac{5}{3}\left(v_{22}-v_{2}^{2}-\frac{3}{5} v_{3}^{2}\right)^{2}-\frac{5}{3} v_{1}^{4}-\frac{5}{3} v_{2}^{4}-\frac{6}{5} v_{3}^{4}\right. \\
& \left.\quad-2 v_{1}^{2} v_{3}^{2}-2 v_{2}^{2} v_{3}^{2}+\frac{5}{3}|D v|^{4}+2|D v|^{2} v_{3}^{2}+\left(v_{2}^{2}+v_{3}^{2}\right)\left(v_{1}^{2}+v_{3}^{2}\right)\right] v_{113} v_{223} \\
\geq & 4 v_{2}^{2} v_{1}^{2} v_{113} v_{223} .
\end{aligned}
$$

If $v_{1} v_{2} \neq 0$, we have $v_{123}^{2} \gtrsim v_{113} v_{223}$. Combining this with (4.19) and (4.20), we get (4.5). If $v_{1} v_{2}=0$, then $F^{12}=-v_{21}+v_{2} v_{1}=0$, putting this into (4.14), we have $F^{11} v_{113}+F^{22} v_{223} \sim 0$, from which we know

$$
-v_{113} v_{223} \sim \frac{F^{11}}{F^{22}} v_{113}^{2} \geq 0 \text {, }
$$

which also implies (4.5).

Case 2: $r_{0}=1$. In this case, $P=\sigma_{2}\left(D^{2} v\right)$ and like case 1 , there exists a positive constant $c_{2}>0$ such that $v_{11} \geq c_{2}>0$. Since $D^{2} v$ is diagonal at $x$, we have

$$
0 \leq v_{33} \leq v_{22} \leq v_{22}+v_{33}+\frac{v_{22} v_{33}}{v_{11}}=\frac{P}{v_{11}} \leq \frac{P}{c_{2}}
$$

i.e.

$$
v_{33} \sim v_{22} \sim 0 \sim P
$$


Differentiating $P$ with respect to $x_{i}$

$$
v_{22 i}+v_{33 i} \sim P_{i} \sim 0 .
$$

Using the above fact and differentiating $P$ with respect to $x_{i}$ and $x_{j}$, we have

$$
P_{i j} \sim-2 v_{12 i} v_{12 j}-2 v_{13 i} v_{13 j}-2 v_{23 i} v_{23 j}+v_{11}\left(v_{22 i j}+v_{33 i j}\right)+\left(v_{22 i} v_{33 j}+v_{22 j} v_{33 i}\right) .
$$

Hence

$$
F^{i j} P_{i j} \sim v_{11} F^{i j}\left(v_{22 i j}+v_{33 i j}\right)-2 F^{i j}\left(v_{12 i} v_{12 j}+2 v_{13 i} v_{13 j}\right)-2 F^{i j}\left(v_{23 i} v_{23 j}+v_{22 i} v_{22 j}\right) .
$$

To handle the first term, we differentiate the equation $F\left(D v, D^{2} v\right)=\lambda$ with respect to $x_{2}$ and $x_{3}$ respectively. We then get

$$
\begin{aligned}
& F^{p_{l}} v_{l 2}+F^{i j} v_{i j 2}=0, \\
& F^{l_{l}} v_{l 22}+F^{i j, r s} v_{i j 2} v_{r s 2}+F^{i j} v_{i j 22} \sim 0, \\
& F^{p_{l}} v_{l 3}+F^{i j} v_{i j 3}=0, \\
& F^{p_{l}} v_{l 33}+F^{i j, r s} v_{i j 3} v_{r s 3}+F^{i j} v_{i j 33} \sim 0 .
\end{aligned}
$$

Adding (4.29) to (4.27) we get

$$
F^{i j}\left(v_{22 i j}+v_{33 i j}\right) \sim-F^{i j, r s}\left(v_{i j 2} v_{r s 2}+v_{i j 3} v_{r s 3}\right) .
$$

This together with (4.25) gives

$$
\begin{gathered}
F^{i j} P_{i j} \sim-v_{11} F^{i j, r s}\left(v_{i j 2} v_{r s 2}+v_{i j 3} v_{r s 3}\right)-2 F^{i j}\left(v_{12 i} v_{12 j}+2 v_{13 i} v_{13 j}\right) \\
-2 F^{i j}\left(v_{23 i} v_{23 j}+v_{22 i} v_{22 j}\right) .
\end{gathered}
$$

To proceed, we need the following lemma (see [4]):

Lemma 4.2. Let $v \in C^{3,1}(\Omega)$ be a convex function. Then for any $O \subset \subset \Omega$, there exists a positive constant $C$ depending only on dist $(O, \partial \Omega)$ and $\|v\|_{C^{3,1}(\Omega)}$ such that

$$
\left|v_{i j \alpha}\right| \leq C\left(\sqrt{v_{i i}}+\sqrt{v_{j j}}\right)
$$

for all $x \in O$ and $1 \leq i, j, \alpha \leq 3$.

Using this lemma, Cauchy inequality and (4.22), we get

$$
v_{i j k}^{2} \sim 0, \quad 2 \leq i, j \leq 3 ; 1 \leq k \leq 3 .
$$

On the other hand, from (4.26) and (4.28), we know

$$
F^{i j} v_{i j 2} \sim F^{i j} v_{i j 3} \sim 0 .
$$

Putting (4.33) into (4.34), we get from Cauchy inequality that $\left(F^{11} v_{112}\right)^{2} \sim\left(F^{11} v_{113}\right)^{2} \sim 0$, i.e.

$$
v_{112}^{2} \sim v_{113}^{2} \sim 0 .
$$

Putting (4.33) and (4.35) into (4.31), we get (4.5) from the Cauchy inequality. This completes the proof of the lemma. 


\section{Proof of Theorem 1.2}

In this section, we use the constant rank theorem and the deformation technique to prove Theorem 1.2. We first prove the strict convexity of $v$ when $\Omega$ is a ball. The proof is following the idea of McCuan [28].

Lemma 5.1 (Case in Ball). Let $B_{R}(0)$ be a ball in $R^{3}$ with radius $R>0$. Let $u \in C^{\infty}\left(B_{R}\right) \cap$ $C^{1,1}\left(\overline{B_{R}}\right)$ be the admissible eigenfunction for $(1.1)$ in $B_{R}(0)$. Then $v=-\log (-u)$ is a strictly convex function in $B_{R}(0)$.

Proof. By the uniqueness of solution for (1.1) up to a positive constant, we know the solution $u$ is a radial function. We set

$$
u(x)=\varphi(|x|)=\varphi(r) \quad \text { for } r=|x| .
$$

where $r \in[0, R]$, then $\varphi<0$ for $r \in[0, R)$ and $\varphi^{\prime}(0)=\varphi(R)=0$.

A straightforward calculation yields

$$
u_{i j}=\left(\varphi^{\prime \prime} r^{-2}-\varphi^{\prime} r^{-3}\right) x_{i} x_{j}+\varphi^{\prime} r^{-1} \delta_{i j}
$$

and the three eigenvalues of $D^{2} u$ are $\frac{\varphi^{\prime}}{r}, \frac{\varphi^{\prime}}{r}, \varphi^{\prime \prime}$. So, (1.1) can be rewritten as

$$
\frac{5\left(\varphi^{\prime}\right)^{2}}{r^{2}}+\frac{6 \varphi^{\prime} \varphi^{\prime \prime}}{r}+\left(\varphi^{\prime \prime}\right)^{2}=\lambda(-\varphi)^{2} \quad \text { in }[0, R)
$$

Since $v=-\log (-\varphi)$, we have

$$
\varphi^{\prime}=e^{-v} v^{\prime}, \quad \varphi^{\prime \prime}=e^{-v}\left(v^{\prime \prime}-\left(v^{\prime}\right)^{2}\right) .
$$

Putting this into (5.2), we get

$$
5\left(v^{\prime}\right)^{2}+6\left[v^{\prime} v^{\prime \prime}-\left(v^{\prime}\right)^{3}\right] r+\left[\left(v^{\prime}\right)^{4}+\left(v^{\prime \prime}\right)^{2}-2\left(v^{\prime}\right)^{2} v^{\prime \prime}\right] r^{2}=\lambda r^{2} \quad \text { in }(0, R) .
$$

Since $\Delta u>0$ in $B_{R}(0)$, i.e.

$$
\left(\frac{\varphi^{\prime}}{r}, \frac{\varphi^{\prime}}{r}, \varphi^{\prime \prime}\right) \in \Gamma_{1}, \quad r \in[0, R)
$$

and $\varphi^{\prime}(0)=0$, we have $\varphi^{\prime \prime}(0)>0$. Thus

$$
v^{\prime \prime}(0)=e^{v} \varphi^{\prime \prime}(0)+\left(v^{\prime}\right)^{2}(0)>0
$$

If there exists some point $r_{1} \in[0, R)$ such that $v^{\prime \prime}\left(r_{1}\right) \leq 0$, we let

$$
r_{0}=\inf \left\{r_{1} \in[0, R) \mid v^{\prime \prime}\left(r_{1}\right) \leq 0\right\} .
$$

Then, $r_{0}>0$ by (5.6) and $v^{\prime \prime}\left(r_{0}\right)=0$. Differentiating (5.4) and taking value at $r_{0}$, we get

$$
-6\left(v^{\prime}\right)^{3}+6 r v^{\prime} v^{\prime \prime \prime}+2 r\left(v^{\prime}\right)^{4}-2 r^{2}\left(v^{\prime}\right)^{2} v^{\prime \prime \prime}=2 \lambda r .
$$


Since $v^{\prime \prime}\left(r_{0}\right)=0$, we know from (5.4) that

$$
5\left(v^{\prime}\right)^{2}-6\left(v^{\prime}\right)^{3} r+\left(v^{\prime}\right)^{4} r^{2}=\lambda r^{2},
$$

i.e.

$$
2 r\left(v^{\prime}\right)^{4}=12\left(v^{\prime}\right)^{3}-\frac{10\left(v^{\prime}\right)^{2}}{r}+2 \lambda r .
$$

Putting (5.10) into (5.8) to substitute $2 r\left(v^{\prime}\right)^{4}$, we have

$$
v^{\prime \prime \prime}\left(r_{0}\right)=\frac{-3\left(v^{\prime}\right)^{2}+\frac{5 v^{\prime}}{r}}{3 r-r^{2} v^{\prime}} .
$$

This implies that $v^{\prime \prime \prime}\left(r_{0}\right)$ has the same sign as $3\left(r v^{\prime}\right)^{3}-14\left(r v^{\prime}\right)^{2}+15 r v^{\prime}$. We also notice that $\varphi^{\prime \prime}\left(r_{0}\right)=e^{-v}\left(v^{\prime \prime}-\left(v^{\prime}\right)^{2}\right)\left(r_{0}\right) \leq 0$ for $v^{\prime \prime}\left(r_{0}\right)=0$, hence by $(5.5)$, we have $\varphi^{\prime}\left(r_{0}\right)>0$ which implies $v^{\prime}\left(r_{0}\right)=e^{v} \varphi^{\prime}\left(r_{0}\right)>0$. So, $v^{\prime \prime \prime}\left(r_{0}\right)$ has the same sign as

$$
3\left(r v^{\prime}\right)^{2}-14\left(r v^{\prime}\right)+15
$$

which means that $v^{\prime \prime \prime}\left(r_{0}\right)>0$ if $r v^{\prime}\left(r_{0}\right) \in\left(-\infty, \frac{5}{3}\right) \cup(3,+\infty)$.

On the other hand, from (5.9), we know

$$
5\left(r v^{\prime}\right)^{2}-6\left(r v^{\prime}\right)^{3}+\left(r v^{\prime}\right)^{4}=\lambda r^{4}>0 .
$$

Hence $r v^{\prime}\left(r_{0}\right) \in(0,1) \cup(5,+\infty) \subset\left(-\infty, \frac{5}{3}\right) \cup(3,+\infty), v^{\prime \prime \prime}\left(r_{0}\right)>0$. However, this contradicts to the fact that $v^{\prime \prime}(r)>0$ for $0 \leq r<r_{0}$ and $v^{\prime \prime}\left(r_{0}\right)=0$. So we have $v^{\prime \prime}(r)>0$ for $0 \leq r<R$, i.e. $v$ is strictly convex in $B_{R}(0)$.

Now, we state the following well-known boundary convexity lemma, see for example Caffarelli-Friedman [6](p. 450, Lemma 4.3) or Korevaar [22] (p. 610, Lemma 2.4).

Lemma 5.2. Let $\Omega \subset R^{3}$ be smooth, bounded and strictly convex. Let $u \in C^{\infty}(\Omega) \cap C^{1,1}(\bar{\Omega})$ satisfies

$$
u<0 \text { in } \Omega, \quad u=0 \text { and } D u \cdot v>0 \text { on } \partial \Omega,
$$

where $v$ is the exterior normal to $\partial \Omega$. Let

$$
\Omega^{\varepsilon}=\{x \in \Omega \mid d(x, \partial \Omega)>\varepsilon\}
$$

and let $v=f(u)$. Then for small enough $\varepsilon>0$ the function $v$ is strictly convex in a boundary strip $\Omega-\Omega^{\varepsilon}$ if $f$ satisfies

$$
\text { (i) } f^{\prime}>0, \quad \text { (ii) } f^{\prime \prime}>0, \quad \text { (iii) } \lim _{u \rightarrow 0^{-}} \frac{f^{\prime}}{f^{\prime \prime}}=0
$$


Proof. The proof can be found in Korevaar [22]. We only point out that in Korevaar [22], $u$ is assumed to be in $C^{2}(\bar{\Omega})$. But we can follow the calculations there to show that the similar result is true for our case when $u \in C^{\infty}(\Omega) \cap C^{1,1}(\bar{\Omega})$. In fact, we know firstly that $u$ has bounded second derivatives a.e. in $\bar{\Omega}$ since $u \in C^{1,1}(\bar{\Omega})$. Following the proof in [22], we then only need to get a lower bound for $\frac{\partial u}{\partial v}$ on $\partial \Omega$. However, this is known from Lemma 3.1. In fact, from (3.12) we know there exists $x_{0} \in \Omega$, positive constants $R$ and $\delta$ such that

$$
u(x) \leq-2 \delta, \quad x \in B_{2 R}\left(x_{0}\right) .
$$

Then, Lemma 3.1 implies the desired result.

Now, we use the deformation technique and the constant rank theorem to prove Theorem 1.2 as in Korevaar-Lewis [23] and Ma-Xu [26, 27].

Proof. If $\Omega$ is the ball $B_{R}(0)$, by Lemma 5.1 , we know $v=-\log (-u)$ is strictly convex in $B_{R}(0)$. For an arbitrary bounded strictly convex domain $\Omega$, set

$$
\Omega_{t}=(1-t) B_{R}(0)+t \Omega, 0 \leq t \leq 1
$$

Then, from the theory of convex bodies, we can deform $B_{R}(0)$ continuously into $\Omega$ by the family $\left\{\Omega_{t}\right\}, 0 \leq t<1$, of strictly convex domain in such a way that $\partial \Omega_{t} \rightarrow \partial \Omega_{s}$ as $t \rightarrow s$ in the sense of Hausdorff distance, whenever $0 \leq s \leq 1$. And the deformation also is chosen so that $\partial \Omega_{t}, 0 \leq t<1$, can be locally represented for some $\alpha, 0<\alpha<1$, by a function whose norm in the space $C^{2, \alpha}$ of functions with Holder continuous second derivatives depends only on $\delta$, whenever $0<t \leq \delta<1$.

Suppose $u_{t} \in C^{\infty}\left(\Omega_{t}\right) \cap C^{1,1}\left(\bar{\Omega}_{t}\right)$ is the admissible solution of $(1.1), v_{t}:=-\log \left(-u_{t}\right)$ and $H_{t}$ is the corresponding hessian matrix of $v_{t}$. First, $H_{0}$ is positive definite, and from Lemma 5.2 we have $H_{\delta}$ is positive definite in an $\varepsilon$ neighborhood of $\partial \Omega_{\delta}$. From Theorem 2.1 and 2.2, we know this bound depends only on the uniformly bounded geometry of $\Omega_{t}$ which depends on the geometry $\Omega$ and $t$. We conclude that if $v(., s)$ is strictly convex for all $0 \leq s<t$, then $v(., t)$ is convex.

So if for some $\delta, 0<\delta<1, H_{\delta}$ is positive semi-definite but not positive definite in $\Omega_{\delta}$, we say it is impossible by constant rank theorem and Lemma 5.2. We conclude $H_{\delta}$ is positive definite. Then $v=-\log (-u)$ is strictly convex in $\Omega$.

\section{Acknowledgments}

The research of the author is supported in partially by the National Natural Science Foundation of China under Grant 11721101. The author would like to thank Prof. Xi-Nan Ma for his encouragement and helpful discussions. 


\section{References}

[1] T. Aubin, Équations de Monge-Ampère réelles. J. Funct. Anal., 41 (3) (1981), 354-377.

[2] T. Aubin, Nonlinear analysis on manifolds. Monge-Ampère equations. Grundlehren der Mathematischen Wissenschaften [Fundamental Principles of Mathematical Sciences], 252. Springer-Verlag, New York, 1982.

[3] H. Berestycki, L. Nirenberg and S.R.S. Varadhan, The principal eigenvalue and maximum principle for second-order elliptic operators in general domains, Comm. Pure Appl. Math., 47(1) (1994), 47-92.

[4] B.J. Bian and P.F. Guan, A microscopic convexity principle for nonlinear partial differential equations, Invent. Math., 177(2) (2009) 307-335.

[5] H.J. Brascamp and E.H. Lieb, On extensions of the Brunn-Minkowski and Prékopa-Leindler theorems, including inequalities for log concave functions, and with an application to the diffusion equation, J. Functional Analysis, 22(4) (1976), 366-389.

[6] L.A. Caffarelli and A. Friedman, Convexity of solutions of semilinear elliptic equations, Duke Math. J., 52(2) (1985), 431-456.

[7] L.A. Caffarelli, L. Nirenberg and J. Spruck, The Dirichlet problem for nonlinear secondorder elliptic equations, III. Functions of the eigenvalues of the Hessian, Acta Math. 155(3-4) (1985), 261-301.

[8] L.A. Caffarelli, P.F. Guan and X.N. Ma, A constant rank theorem for solutions of fully nonlinear elliptic equations, Comm. Pure Appl. Math., 60(12) (2007), 1769-1791.

[9] L.C. Evans, Partial differential equations. Second edition, Graduate Studies in Mathematics, 19. American Mathematical Society, Providence, RI, 2010.

[10] J.X. Fu, Z.Z. Wang and D.M. Wu, Form-type Calabi-Yau equations, Math. Res. Lett., 17(5) (2010), 887-903.

[11] J.X. Fu, Z.Z. Wang and D.M. Wu, Form-type equations on Kähler manifolds of nonnegative orthogonal bisectional curvature. Calc. Var. Partial Differential Equations 52(1-2) (2015), 32344 .

[12] D. Geng, Q.Y. Yu and C.Z. Qu, The eigenvalue problem for Hessian operators, Nonlinear Anal., 25(1) (1995), 27-40.

[13] D. Gilbarg and N.S. Trudinger, Elliptic Partial Differential Equations of Second Order. Reprint of the 1998 edition, Classics in Mathematics. Springer-Verlag, Berlin, 2001.

[14] P.F. Guan and X.N. Ma, The Christoffel-Minkowski problem, I. Convexity of solutions of a Hessian equation, Invent. Math., 151(3) (2003), 553-577.

[15] P.F. Guan, X.N. Ma and F. Zhou, The Christofel-Minkowski problem, III. Existence and convexity of admissible solutions, Comm. Pure Appl. Math., 59(9) (2006), 1352-1376.

[16] F. Han, X.N. Ma and D.M. Wu, A constant rank theorem for Hermitian k-convex solutions of complex Laplace equations, Methods Appl. Anal., 16(2) (2009), 263-289.

[17] F. Han, X.N. Ma and D.M. Wu, The existence of $k$-convex hypersurface with prescribed mean curvature, Calc. Var. Partial Differential Equations, 42(1-2) (2011), 43-72.

[18] F.R. Harvey and H.B.J. Lawson, Dirichlet duality and the nonlinear Dirichlet problem, Comm. Pure Appl. Math., 62(3) (2009), 396-443.

[19] F.R. Harvey and H.B.J. Lawson, Geometric plurisubharmonicity and convexity: an introduction, Adv. Math., 230(4-6) (2012), 2428-2456.

[20] F.R. Harvey and H.B.J. Lawson, P-convexity, p-plurisubharmonicity and the Levi problem, Indiana Univ. Math. J., 62(1) (2013), 149-169.

[21] J.X. Hong, G.G. Huang and W.Y. Wang, Existence of global smooth solutions to Dirichlet 
problem for degenerate elliptic Monge-Ampere equations, Comm. Partial Differential Equations, 36(4) (2011), 635-656.

[22] N.J. Korevaar, Convex solutions to nonlinear elliptic and parabolic boundary value problems, Indiana Univ. Math. J., 32(4) (1983), 603-614.

[23] N.J. Korevaar and J.L. Lewis, Convex solutions of certain elliptic equations have constant rank Hessians, Arch. Rational Mech. Anal., 97(1) (1987), 19-32.

[24] N.Q. Le and O. Savin, Schauder estimates for degenerate Monge-Ampère equations and smoothness of the eigenfunctions, Invent. Math. 207(1) (2017), 389-423.

[25] P.L. Lions, Two remarks on Monge-Ampère equations, Ann. Mat. Pura Appl., 142(4) (1985), 263-275.

[26] P. Liu, X.N. Ma and L. Xu, A Brunn-Minkowski inequality for the Hessian eigenvalue in three-dimensional convex domain. Adv. Math., 225(3) (2010), 1616-1633.

[27] X.N. Ma and L. Xu, The convexity of solution of a class Hessian equation in bounded convex domain in $\mathbb{R}^{3}$, J. Funct. Anal., 255(7) (2008), 1713-1723.

[28] J. McCuan, Concavity, quasiconcavity, and quasilinear elliptic equations, Taiwanese J. Math., 6(2) (2002), 2, 157-174.

[29] R. Schneider, Convex Bodies: the Brunn-Minkowski Theory. Second expanded edition, Encyclopedia of Mathematics and its Applications, 151. Cambridge University Press, Cambridge, 2014.

[30] I.M. Singer, B. Wong, S.T. Yau nad S.S.-T. Yau, An estimate of the gap of the first two eigenvalues in the Schrödinger operator. Ann. Scuola Norm. Sup. Pisa Cl. Sci. (4) 12 (1985), no. 2, 319-333.

[31] V. Tosatti, B. Weinkove, The Monge-Ampère equation for $(n-1)$-plurisubharmonic functions on a compact Kähler manifold, J. Amer. Math. Soc., 30(2) (2017), 311-346.

[32] X.J. Wang, Existence of multiple solutions to the equations of Monge-Ampère type, J. Differential Equations, 100(1) (1992), 95-118.

[33] X.J. Wang, A class of fully nonlinear elliptic equations and related functionals, Indiana Univ. Math. J. 43(1) (1994), 25-54.

[34] X.J. Wang, The $k$-Hessian Equations, Geometric Analysis and PDEs, 177-252, Lecture Notes in Math., 1977, Springer, Dordrecht, 2009. 